\title{
Perceptions of Research Participation among a Sample of Florida Residents Aged 50 and Over Reporting Dementia
}

\author{
Sadaf Arefi Milani ${ }^{1}$ D $\cdot$ Linda B. Cottler $^{2} \cdot$ Catherine W. Striley $^{2}$
}

Accepted: 25 July 2021 / Published online: 31 August 2021

(C) The Author(s), under exclusive licence to Springer Science+Business Media, LLC, part of Springer Nature 2021

\begin{abstract}
As the population ages, the prevalence of dementia will increase. More research is needed; however, low rates of research participation, especially by older adults, hinder progress. Data came from HealthStreet, a University of Florida community engagement program in which community health workers assess community members for their health conditions. Adults 50 and over were included in these analyses; their perceptions of health research studies were assessed. Our sample had an average age of 60.5 years and $4.3 \%$ reported dementia $(n=4,881)$. Overall, older adults reported a high willingness to participate in research. Individuals who reported dementia had 0.64 times the odds of reporting willingness to participate in a research study that required an overnight stay, compared to those who did not report dementia (95\% CI: 0.45-0.89). We report on willingness to participate in research in a more granular basis than has been previously done. Community members reporting dementia, compared to those without, were as or more likely to desire future participation in health research. However, barriers to participation remain and must be addressed.
\end{abstract}

Keywords Dementia $\cdot$ Alzheimer's disease $\cdot$ Research Participation $\cdot$ Community Health

\section{Background}

Including individuals in research on Alzheimer's disease and related dementias (ADRD) is increasingly urgent. Currently, 5.8 million American's are living with Alzheimer's and this number is expected to triple by 2050 (Alzheimer's Association,

Sadaf Arefi Milani

samilani@utmb.edu

1 Department of Internal Medicine, University of Texas Medical Branch, Galveston, TX, USA

2 Department of Epidemiology, College of Public Health and Health Professions \& College of Medicine, University of Florida, Gainesville, FL, USA 
2020). The first objective of the National Plan to Address Alzheimer's disease is to "prevent and effectively treat ADRD by 2025" (U.S. Department of Health and Human Services, 2020). One of the strategies to achieve this goal involves increasing recruitment and enrollment in clinical trials, with an emphasis on underrepresented communities (U.S. Department of Health and Human Services, 2020). Recognizing the importance of increasing enrollment in studies on dementia, the National Institute on Aging (NIA) released a National Strategy for Recruitment and Participation in Alzheimer's and Related Dementias Clinical Research with the goal of engaging the public in ADRD research (Elliott, 2020). However, this is a big challenge. Currently, over 250 clinical trials on AD in the United States are recruiting (US National Library of Medicine, 2021). In 2018, almost 200 clinical trials were seeking to enroll over 270,000 participants (Elliott, 2020). The number of individuals needed to screen for studies is much higher, because only about $10 \%$ of screened individuals are eligible to participate after screening (Watson et al., 2014).

Older adults, especially racial/ethnic minorities, are underrepresented in research (McMurdo et al., 2011; Mody et al., 2008). Our previous work using data from HealthStreet, a community engagement program at the University of Florida, has found that, overall, community-dwelling adults aged 50 and older are willing to participate in research, but report low past research participation (Milani et al., 2021). Previous research has found that older people want to participate in research because of a combination of altruism and self-interest, but barriers to their participation remain and need to be addressed (Grill \& Karlawish, 2010; Jefferson et al., 2011; Witham \& McMurdo, 2007). Non-geriatrician subspecialists who conduct research on aging also report challenges to including older adults in research, such as a lack of training in aging research and a lack of knowledge of high-priority research questions (Barrett Bowling et al., 2019). They also reported that their study structure may be too burdensome for older adults, as protocols may require multiple in-person visits, and reported concerns about the high potential for reporting adverse events and high withdrawal rates (Barrett Bowling et al., 2019).

Clinical trials on ADRD face the common challenges of all clinical trials, but also have unique challenges specific to ADRD trials (Elliott, 2020). One challenge is stringent study criteria. Less than $30 \%$ of ADRD patients are eligible for clinical trials (Grill \& Galvin, 2014). These individuals may be excluded because of advanced age, comorbid conditions, multiple medications, cogitative difficulties, and lack of transportation to the study site (Grill \& Galvin, 2014; Grill \& Karlawish, 2010; Jefferson et al., 2011; Witham \& McMurdo, 2007). Another challenge is a lack of awareness of clinical trials. Primary care physicians may not always detect cognitive impairment and they might be unaware of local research opportunities (Elliott, 2020; Watson et al., 2014). Additionally, many studies may require the participation of a study partner, who are usually spouses, but many older adults are unmarried or live alone (Elliott, 2020). Unimpaired volunteers are also needed, as AD pathology begins much earlier than the onset of symptoms (Elliott, 2020; Holtzman et al., 2011). This is especially important in trials that test prevention interventions, as these require at-risk, unimpaired individuals who are willing to undergo screening (Watson et al., 2014). 
Low participation rates in research are hindering progress; there is a need for more participation in research from individuals with dementia to make strides towards understanding this disease (Fargo et al., 2016). The Community Health Worker Model could help engage more individuals with dementia into research. Community health workers (CHWs) are lay community members who are trained to deliver education and interventions, and who can act as an intermediary between communities and researchers (Cottler, Striley, et al., 2013; Landers \& Stover, 2011); they can provide an important extension to community members who may not otherwise participate in research studies.

The analyses here describe the willingness of community members who report a history of dementia to participate in health research studies, on a more granular basis that has been previously done. We report on attitudes towards participating in very specific types of health research, including research with no remuneration, research where the person has to stay overnight, and research where medicine must be taken. We hypothesize that individuals who self-report dementia will be as likely or more likely to be willing to participate in research studies compared to individuals not reporting dementia. Understanding their willingness to participate in research, as well as their trust in research and researchers, is critical to the field.

\section{Methods}

\section{Population}

Participants were members of HealthStreet, a community engagement program at the University of Florida which uses CHWs to assess the health conditions and concerns of community members. CHWs were trained and certified in introducing the Montreal Cognitive Assessment (MoCA), administering and scoring the MoCA, and making appropriate referrals based on the original MoCA cut point by a licensed psychologist, who administers the MoCA, and by Dr. Striley, who is certified to administer the MoCA. All CHWs were tested on their skills before they were allowed in the field, first by the lead CHW and then by Dr. Striley. This was an add on to their regular training and certification process which requires a minimum of $10 \mathrm{~h}$ of classroom training in addition to Health Insurance Portability and Accountability Act (HIPAA) and Collaborative Institutional Training Initiative (CITI) Institutional Review Board (IRB) training, and 20 hours of supervised field work before faculty certify them as ready to work in the field. CHWs engage community members daily where they live, work, and recreate. Individuals are approached at recruitment sites, including recreational parks, local libraries, laundromats, churches, and public events. HealthStreet has a high recruitment yield of $84 \%$ since its start in Florida in October 2011. Once consented [all participants were judged by CHWs to be able to give valid consent] in the community, participants were interviewed by a CHW, using the HealthStreet intake form, a written form used by CHWs. The interview, which took approximately twenty minutes, elicited an individual's demographic information, socioeconomic status, physical and mental health, substance use, and willingness to participate in health research. Interviews were conducted in 
Spanish when appropriate, using bilingual CHWs. After the interview, participants were considered to be HealthStreet members. HealthStreet members can utilize services offered through HealthStreet, including computers for public use, health education classes, support groups, clothes and toiletry pantry, on-site free health care, mental health care through safety net provider clinics, and blood pressure checks at the physical HealthStreet location. HealthStreet also acts as a liaison between community members and the research community. A HealthStreet Study Navigator maintains a database of studies at the University of Florida, including their selection criteria. Participants interested in participating in research who are deemed eligible, based on the information provided during their interview, are contacted for recruitment.

This analysis was limited to HealthStreet participants aged 50 and over who completed the HealthStreet Intake Form from November 2011 to December 2020 $(n=4,881)$. Of these, $15.0 \%(n=800)$ of participants were recruited using targeted recruiting for older individuals to be included in studies on Alzheimer's disease.

\section{Measures}

Independent variable: Participants were asked: "have you ever been told you have dementia?" with yes, no, or refuse response options. Individuals who responded yes were categorized as having dementia. Individuals aged 50 and over were included in this analysis.

Dependent variable: The outcomes of this study were: 1) expressed willingness to participate in different types of research studies and 2) trust in research and researchers. The HealthStreet Intake Form assesses past health research participation. It elicits information on willingness to participate in research overall, and then elicits the respondent's willingness to be a volunteer in 8 different types of research (Table 2).

Covariates: Covariates included were age, gender, race/ethnicity, marital status, education level, insurance status, food insecurity, hypertension, diabetes, and depression, all elicited through the HealthStreet Intake Form. Age was recorded as decade of age and gender was coded as either male or female. Race/ethnicity was recorded as non-Hispanic White, non-Hispanic Black, non-Hispanic Other, or Hispanic. Marital status was coded as not married (never married, separated, widowed, or divorced) or currently married. The HealthStreet Intake Form contains the question "have there been times in the last 12 months when you did not have enough money to buy food that you or your family needed" providing the basis for our food insecurity variable, which was coded as yes/no. "Last grade completed?" was categorized as less than 12 years vs holding a GED or completing high school ( $\geq 12$ years). Self-reported lifetime hypertension, diabetes, and depression were coded as yes/no variables.

\section{Data Analysis}

SAS $®$ software version 9.4 was used for the analysis. Individuals who were missing a response for the lifetime dementia variable were not included in this analysis 
$(\mathrm{n}=26 ; 0.48 \%)$. Chi-square test of independence, analysis of variance tests, and logistic regression were used to test between group differences by dementia status. P-values less than 0.05 were considered to be statistically significant. Multivariable logistic regression models were used to assess the odds of willingness to participate in research for those research types that had a bivariate association, adjusting for age gender, race/ethnicity, marital status, education level, insurance status, food insecurity, hypertension, diabetes, and depression.

\section{Results}

Of the 4,881 respondents, 209 (4.3\%) reported being told they had dementia (Table 1). Some differences between groups were noted: those reporting dementia were older, more often male, non-Hispanic White, not married, insured, and food insecure; they also reported more diabetes and depression. Almost $60 \%$ of participants reporting dementia also reported depression. No differences between groups were noted for educational level or hypertension.

As shown in Table 2, older adults in general are likely to want to participate in all types of research. In fact, very high proportions of adults $50+$, regardless of dementia status, reported that they would be likely to participate in studies that only asked about their health ( $>94 \%)$, that wanted to look at medical records (from $88.1 \%$ to $90.4 \%$ ), that needed a blood or a genetic sample ( $88.1 \%$ to $90.0 \%$ ), that required participants to take medicine $(63.2 \%$ to $66.7 \%)$, that tested medical equipment (88.3\% to $90.4 \%$ ), or that provided no compensation (80.3\% to $85.7 \%$ ). Significantly fewer participants reporting dementia vs those not, reported being willing to participate in studies that required an overnight stay in a hospital or clinic $(73.7 \%$ vs $79.5 \%)$. After adjustment for age, gender, race, employment status, and self-reported depression, those who reported dementia were 0.64 times less likely than participants not reporting dementia to report being willing to participate in a research study that required an overnight stay $(95 \%$ Confidence Interval: 0.45-0.89) (data not shown).

Participants reported similar interest in participating in research, regardless of dementia status. About $55.5 \%$ of participants reporting dementia and $54.6 \%$ of participants not reporting dementia said they were definitely interested in participating in research.

Participants reporting dementia cited a significantly lower remuneration as a fair amount for participation in a study that lasted about an hour and a half and involved an interview and a blood test, compared to those not reporting dementia $(\$ 60.2 \pm 87.1$ vs $\$ 88.91 \pm 159.1)$. On a scale of one to ten, where ten is complete trust, individuals, regardless of dementia status, reported high trust in research and researchers (Table 2). Those reporting dementia rated their trust in research as 7.7 [standard deviation (SD): 7.0], while those who did not report dementia rated their trust as 8.4 (SD: 9.7$), p=0.1157$. Those reporting dementia rated their 
Table 1 Sociodemographic characteristics and comorbid diseases of HealthStreet participants 50 years of age and older by dementia status $(n=4,881)$

$\begin{array}{lll}\text { Participants Reporting } & \text { Participants not Reporting } & \text { P-Value } \\ \text { Dementia } & \text { Dementia } \\ (n=209 ; 4.3 \%) & (n=4,672 ; 95.7 \%)\end{array}$

Sociodemographic Characteristics

Age

50-59

60-69

70-79

$80+$

Gender

Male

Female

Race/ethnicity

Non-Hispanic White

Non-Hispanic Black

Non-Hispanic Other

Hispanic

Marital Status

Currently Married

Not Married

Widowed

Education level

Less than high school

At least GED/high school

Health Insurance Status

Insured

Uninsured

Food Insecurity

Yes

No

Chronic Diseases

Hypertension

Yes

No

Diabetes

Yes

No

Depression

Yes

No
$69(33.0 \%)$
$66(31.6 \%)$
$53(25.4 \%)$
$21(10.1 \%)$

$110(52.6 \%)$

$99(47.4 \%)$

$134(64.1 \%)$

$60(28.7 \%)$

$5(2.4 \%)$

$10(4.8 \%)$

$56(26.8 \%)$

$112(53.6 \%)$

$41(19.6 \%)$

$35(16.8 \%)$

$174(83.3 \%)$

$177(84.7 \%)$

$32(15.3 \%)$

$119(56.9 \%)$

$90(43.1 \%)$

$121(57.9 \%)$

$88(42.1 \%)$

$62(29.7 \%)$

$147(70.3 \%)$

$124(59.3 \%)$

$85(40.7 \%)$
$<0.0001$

$2425(51.9 \%)$

$1579(33.8 \%)$

$540(11.6 \%)$

$128(2.7 \%)$

1931 (41.3\%)

$2741(58.7 \%)$

$1873(40.1 \%)$

$2453(52.5 \%)$

$106(2.3 \%)$

$240(5.1 \%)$

0.0055

$1249(26.7 \%)$

$2850(61.0 \%)$

$573(12.3 \%)$

0.9961

$783(16.8 \%)$

$3889(83.2 \%)$

$3310(70.9 \%)$

$1362(29.2 \%)$

0.0060

2207 (47.2\%)

$2465(52.8 \%)$

$2511(53.8 \%)$

$2161(46.3 \%)$

1065 (22.8\%)

3607 (77.2\%)

$1488(31.9 \%)$

$3184(68.2 \%)$ 


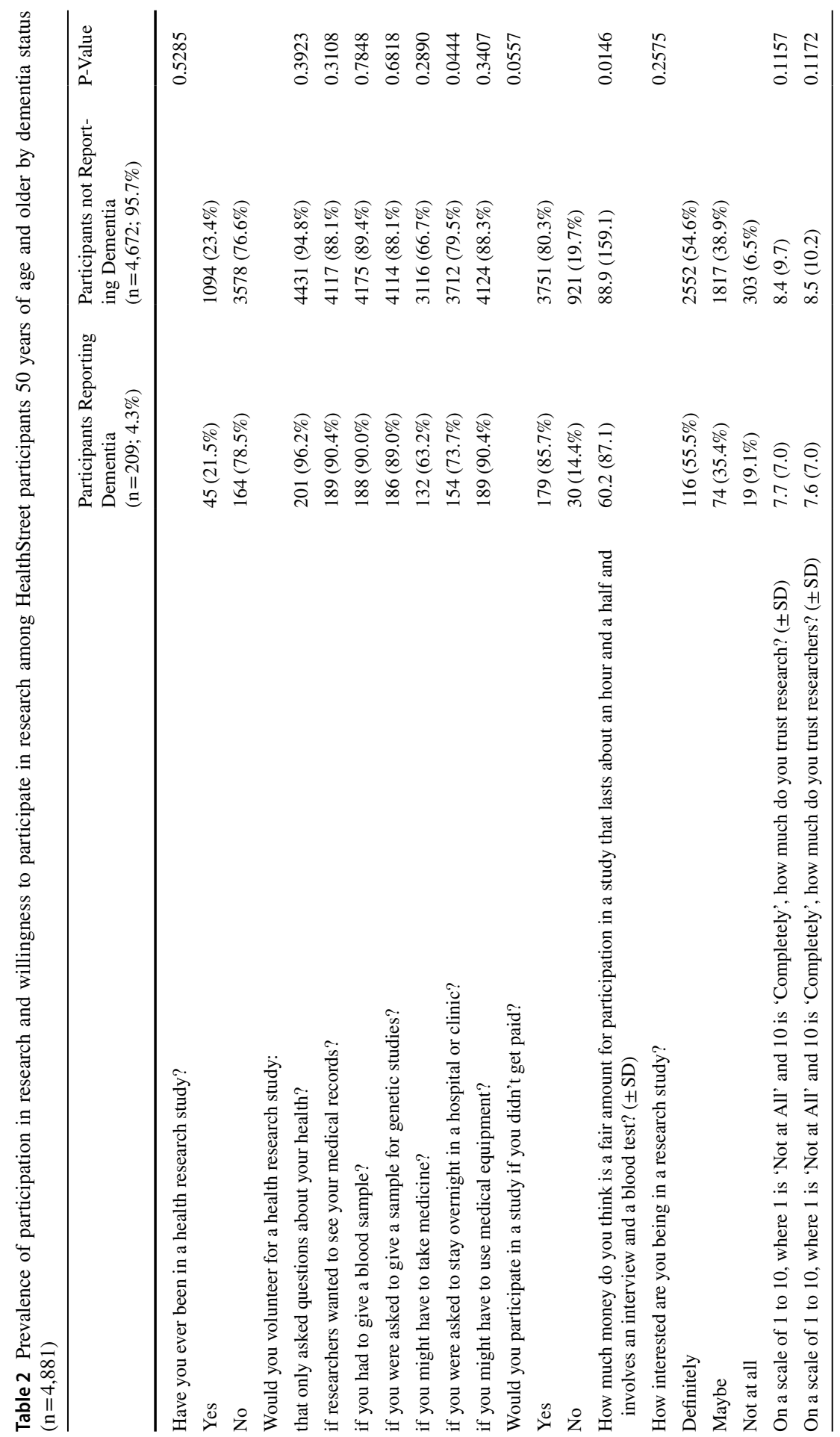


trust in researchers at 7.6 (SD: 7.0), while those who did not report dementia rated their trust as 8.5 (SD: 10.2$)(\mathrm{p}=0.1172)$.

\section{Discussion}

In this study of older community-dwelling adults aged 50 and older, we report on attitudes towards research in a more granular basis than has been previously done. We report on attitudes towards participating in very specific types of health research, including research with no remuneration, research where the person has to stay overnight, and research where medicine must be taken. This level of granularity allows us to better understand, not only the likelihood of older adults with possible dementia to participate in much needed research on dementia, but also their likelihood based on specific interests and research characteristics. This information can help researchers tailor their study designs to overcome barriers in the design stage and help research teams address barriers to recruitment during the conduct of the study. Additional site-specific qualitative research can be used to develop an even more nuanced understanding of attitudes towards specific elements of research from the potential participants' point of view.

Older adults in our sample reported low rates of participation in health research overall, with no differences by dementia status; however, they did report higher participation rates than others have reported. Previous research has found that $10.1 \%$ to $25.9 \%$ of community members report past participation in health research studies in communities where research intensive universities are in close proximity (Cottler, McCloskey, et al., 2013) and this group of community members' past participation falls within this range. However, older adults are more underrepresented in research compared to younger adults and less likely to have previously participated in research. Since the sample was drawn from a sample living near a research-intensive university, these community members may have been more likely to be exposed to and participate in research (Cottler, McCloskey, et al., 2013).

A high percentage of all participants were interested in participating in health research studies but reported low past research participation. Regardless of self-reported dementia status, participants reported high trust in research and researchers. Previous work has found that distrust of researchers, especially among non-White populations, hinders research participation (Smirnoff et al., 2018). The high trust reported by our participants may be attributable to the use of CHWs, who are trusted members of the community they serve. This demonstrates that CHWs may be an effective way to build trust with the community and engage community members with opportunities to participate in research. In particular, during the COVID-19 pandemic, when older adults might have been more isolated, it is very important to engage them and build trust so all individuals can feel comfortable participating in research.

Over $90 \%$ of individuals reporting dementia in this study were interested in participating in research. This may be due to the fact that there is no cure for dementia, so individuals with dementia may be more willing to take medicine 
in hopes of treatment that could potentially cure, reduce symptoms, or reduce impairment (Howe, 2012). Individuals with dementia might also be willing to participate because of altruistic intent with the goal of preventing others from suffering with the disease in the future (Howe, 2012; Knebl \& Patki, 2010). However, individuals reporting dementia were almost $40 \%$ less likely to be willing to participate in a research study that required an overnight stay. This requirement may be seen as inconveniencing or burdensome. Individuals with dementia more often reported depression or diabetes, consistent with the findings of previous literature (Alzheimer's Association, 2020; Cheng et al., 2012; Richard et al., 2013). This double burden may have caused these older adults to utilize or need to utilize health services frequently and may have also increased their desire to contribute to research. These findings have face validity; individuals with dementia who are competent enough to complete the interview answer the questions consistently with how we would expect. It is important to note that these individuals were not deemed as impaired by the interviewing CHWs.

A previous study, among adults aged 50 and older enrolled in a communitybased research recruitment registry, examined willingness to be contacted about AD research opportunities (Salazar, Hoang, Gillen, \& Grill, 2020). This study explored willingness to participate in research, looking at specific types of AD study, but did not compare willingness by dementia status. Instead, they were interested in racial/ethnic differences; however, $16.2 \%$ of their overall sample reported some neurological disorder (Salazar et al., 2020). Despite this difference in study design, our findings are comparable. Salazar et al. observed, across all racial and ethnic groups, the willingness to be contacted about all studies, except those that require a lumbar puncture or autopsy, was greater than $70 \%$ (Salazar et al., 2020). For our study, willingness to participate in all research types was greater than $70 \%$, except for studies that require participants to take medication, where interest was $63.2 \%$ for those reporting dementia and $66.7 \%$ for those not reporting dementia. This difference may be attributable to differences in question wording. Salazar and colleagues asked participants about their willingness to be contacted for different types of research, while we inquired about participants' willingness to participate in different types of research.

This study is among the first to look at past self-reported research participation and attitudes towards participation in individuals with dementia. Such research is especially important since these participants were community members who may or may not be taking medications or otherwise involved in studies. Half of the individuals reporting dementia in our sample were non-Hispanic Black and the other half were primarily non-Hispanic White; five percent were Hispanic. Non-Hispanic Black and Hispanic individuals have a larger burden of dementia in the United States population (Alzheimer's Association, 2020; Chin et al., 2011; Gurland et al., 1999). The fact that minority members with dementia are interested in participating in research is important; the National Institute of Aging is prioritizing including minority populations in research to reduce enduring health disparities in AD. Consistent with previous studies, this analysis found that minority populations are willing to participate in research; barriers to their participation need to be identified to reduce health disparities in this disease (Ceballos 
et al., 2014; Cottler, McCloskey, et al., 2013; Milani et al. 2021; Wendler et al., 2005).

\section{Limitations}

A few limitations should be noted. These analyses are part of an ongoing community engagement program with the goal of taking the pulse of the community. Therefore, we did not distinguish between types of dementia, but focused on self-reported dementia as a whole. The reliability of self-reported dementia may be questioned. However, CHWs have started administering the MoCA (Nasreddine et al., 2005) to older community members; $673(13.8 \%)$ of those included in these analyses have completed a MoCA. Of the 82 individuals reporting dementia who completed a MoCA, 60 (73.2\%) scored below the cut off for cognitive impairment, showing a high rate of congruence with positive self-report $(\mathrm{p}<0.001)$. Of the 598 individuals who did not report dementia who completed a MoCA, more than half $(53.6 \%)$ scored below the cut off for cognitive impairment.

Covariates included in the analysis were also determined by self-report; however, previous literature has found that self-report of disease and medical records generally are in agreement (Johansson et al., 1999; Okura et al., 2004). Moreover, expressed willingness to participate in research may not fully reflect how research is conducted in the real world; individuals participating in clinical trials may be required to do a combination of activities (such as give a blood sample and use medical records). At the time of this analysis, we were recruiting from participants who were judged as able to consent for themselves rather recruiting from populations needing legally authorized representatives (LAR) to consent for them. HealthStreet CHWs seldom encounter people who are severely impaired with a caregiver; it may happen when CHWs visit supported living arrangements, but this is very rare. Thus, we ruled out the inclusion of more impaired individuals. This exclusion may account for the more equal burden of dementia in this high proportion minority sample. The results of this analysis may also be influenced by selection bias, since all individuals included in this analysis already agreed to participate in the HealthStreet program. This is unlikely, however, given the small refusal rate $(13.9 \%)$ and the small ineligibility rate $(1.4 \%)$. The findings of this analysis provide a valuable baseline of the perceptions of research of individuals self-reporting dementia. In the future, we will be able to report how LARs perceptions and attitudes differ from those of individuals who self-report with dementia.

\section{Conclusions}

As the prevalence of dementia is expected to increase, more research will be needed to treat and even cure dementia. More trials equate to the need for more participants. While we found that fewer than one in four individuals reporting dementia had participated in some research, the high interest in participation shows that this is still a largely under-invited and under-recruited population. Community dwelling 
populations are very likely to be willing to participate in research trials, but they need to be invited to do so. Community engagement using CHWs can be an effective way to involve these populations.

Funding This work was supported by the University of Florida Clinical and Translational Science Institute, which is supported in part by the National Institutes of Health's National Center for Advancing Translational Sciences [UL1TR001427], by the Florida Department of Health - Ed and Ethel Moore Alzheimer's Disease Research Program [6AZ05], and by the Department of Epidemiology funding from the College of Medicine and College of Public Health and Health Professions. Dr. Milani was funded by the Graduate School Fellowship at the University of Florida for a portion of this work. Dr. Milani is supported by a research career development award (K12HD052023: Building Interdisciplinary Research Careers in Women's Health Program-BIRCWH; Berenson, PI) from the National Institutes of Health/ Office of the Director (OD)/National Institute of Allergy and Infectious Diseases (NIAID), and Eunice Kennedy Shriver National Institute of Child Health \& Human Development (NICHD). Dr. Cottler and Dr. Striley receive some funding from the 1Florida Alzheimer's Disease Research Center [NIH P50 AG047266], sponsored by the National Institute on Aging which governs Alzheimer's Disease Research Centers through the National Alzheimer's Coordinating Center. The content is solely the responsibility of the authors and does not necessarily represent the official view of the National Institutes of Health.

\section{Declarations}

Conflicts of interest The authors have no conflicts of interest to declare.

Informed Consent Health Street, a community engagement program at the University of Florida, was approved by the University's Institutional Review Board (reference number: IRB201600459) Written and informed consent and obtained from participants.

Ethical Treatment of Experimental Subjects (Animals and Humans) No experimental treatment was conducted on either human or animal subjects in this study.

\section{References}

Alzheimer's Association. 20202020 Alzheimer's disease facts and figures Alzheimer's \& Dementia 163391 460 https://doi.org/10.1002/alz.12068

Bowling, C. B., Whitson, H. E., \& Johnson, T. M., 2nd. (2019). The 5Ts: Preliminary Development of a Framework to Support Inclusion of Older Adults in Research. Journal of the American Geriatrics Society, 67(2), 342-346. https://doi.org/10.1111/jgs.15785

Ceballos, R. M., Knerr, S., Scott, M. A., Hohl, S. D., Malen, R. C., Vilchis, H., \& Thompson, B. (2014). Latino beliefs about biomedical research participation: a qualitative study on the U.S.-Mexico border. Journal of empirical research on human research ethics : JERHRE, 9(4), 10-21. https://doi.org/10. $1177 / 1556264614544454$

Cheng, G., Huang, C., Deng, H., \& Wang, H. (2012). Diabetes as a risk factor for dementia and mild cognitive impairment: A meta-analysis of longitudinal studies. Internal Medicine Journal, 42(5), 484-491. https://doi.org/10.1111/j.1445-5994.2012.02758.x

Chin, A. L., Negash, S., \& Hamilton, R. (2011). Diversity and disparity in dementia: The impact of ethnoracial differences in Alzheimer disease. Alzheimer Disease and Associated Disorders, 25(3), 187-195. https://doi.org/10.1097/WAD.0b013e318211c6c9

Cottler, L. B., McCloskey, D. J., Aguilar-Gaxiola, S., Bennett, N. M., Strelnick, H., Dwyer-White, M, Collyar, D. E., Ajinkya, S., Seifer, S. D., O’Leary, C. C., Striley, C. W., \& Evanoff, B. (2013) Community Needs Concerns and Perceptions About Health Research: Findings From the Clinical and Translational Science Award Sentinel Network. American Journal of Public Health, 103(9), 1685-1692. https://doi.org/ 10.2105/AJPH.2012.300941

Cottler, L. B., Striley, C. W., O'leary, C. C., Ruktanonchai, C. W., \& Wilhelm, K. A. (2013). Engaging the community in research with the HealthStreet model: national and international perspectives. 
In Translational Medicine-What, Why and How: An International Perspective (Vol. 3, pp. 98-109): Karger Publishers.

Elliott, C. L. (2020). Together We Make the Difference: National Strategy for Recruitment and Participation in Alzheimer's and Related Dementias Clinical Research. Ethnicity \& Disease, 30(Suppl), 705-708.

Fargo, K. N., Carrillo, M. C., Weiner, M. W., Potter, W. Z., \& Khachaturian, Z. (2016). The crisis in recruitment for clinical trials in Alzheimer's and dementia: An action plan for solutions. Alzheimer's \& Dementia, 12(11), 1113-1115. https://doi.org/10.1016/j.jalz.2016.10.001

Grill, J. D., \& Galvin, J. E. (2014). Facilitating Alzheimer Disease Research Recruitment. Alzheimer Disease \& Associated Disorders, 28(1), 1-8. https://doi.org/10.1097/wad.0000000000000016

Grill, J. D., \& Karlawish, J. (2010). Addressing the challenges to successful recruitment and retention in Alzheimer's disease clinical trials. Alzheimer's Research \& Therapy, 2(6), 34-34. https://doi.org/10.1186/ alzrt58

Gurland, B. J., Wilder, D. E., Lantigua, R., Stern, Y., Chen, J. M., Killeffer, E. H. P., \& Mayeux, R. (1999). Rates of dementia in three ethnoracial groups. International Journal of Geriatric Psychiatry, 14(6), 481-493.

Holtzman, D. M., Morris, J. C., \& Goate, A. M. (2011). Alzheimer's disease: the challenge of the second century. Science translational medicine, 3(77), 77sr71-77sr71.

Howe, E. (2012). Informed consent, participation in research, and the Alzheimer's patient. Innovations in Clinical Neuroscience, 9(5-6), 47-51.

Jefferson, A. L., Lambe, S., Chaisson, C., Palmisano, J., Horvath, K. J., \& Karlawish, J. (2011). Clinical research participation among aging adults enrolled in an Alzheimer's Disease Center research registry. Journal of Alzheimer's Disease : JAD, 23(3), 443-452. https://doi.org/10.3233/JAD-2010-101536

Johansson, J., Hellénius, M. L., Elofsson, S., \& Krakau, I. (1999). Self-report as a selection instrument in screening for cardiovascular disease risk. American Journal of Preventive Medicine, 16(4), 322-324. https://doi.org/10.1016/s0749-3797(99)00019-7

Knebl, J. A., \& Patki, D. (2010). Recruitment of subjects into clinical trials for Alzheimer disease. Journal of the American Osteopathic Association, 110(9 Suppl 8), S43-49.

Landers, S. J., \& Stover, G. N. (2011). Community health workers-practice and promise. American Journal of Public Health, 101(12), 2198-2198. https://doi.org/10.2105/AJPH.2011.300371

McMurdo, M. E. T., Roberts, H., Parker, S., Wyatt, N., May, H., Goodman, C., ... Age and Ageing Specialty Group, N. C. C. R. N. (2011). Improving recruitment of older people to research through good practice. Age and Ageing, 40(6), 659-665. https://doi.org/10.1093/ageing/afr115

Milan, S. A., Swain, M., Otufowora, A., Cottler, L. B., \& Striley, C. W. (2021) Willingness to Participate in Health Research Among Community-Dwelling Middle-Aged and Older Adults: Does Race/Ethnicity Matter?. Journal of Racial and Ethnic Health Disparities, 8(3), 773-782. https://doi.org/10.1007/ s40615-020-00839-y

Mody, L., Miller, D. K., McGloin, J. M., Freeman, M., Marcantonio, E. R., Magaziner, J., \& Studenski, S. (2008). Recruitment and retention of older adults in aging research. Journal of the American Geriatrics Society, 56(12), 2340-2348. https://doi.org/10.1111/j.1532-5415.2008.02015.x

Nasreddine, Z. S., Phillips, N. A., Bedirian, V., Charbonneau, S., Whitehead, V., Collin, I., \& Chertkow, H. (2005). The Montreal Cognitive Assessment, MoCA: A brief screening tool for mild cognitive impairment. Journal of the American Geriatrics Society, 53(4), 695-699. https://doi.org/10.1111/j.1532-5415. 2005.53221.x

Okura, Y., Urban, L. H., Mahoney, D. W., Jacobsen, S. J., \& Rodeheffer, R. J. (2004). Agreement between self-report questionnaires and medical record data was substantial for diabetes, hypertension, myocardial infarction and stroke but not for heart failure. Journal of Clinical Epidemiology, 57(10), 10961103. https://doi.org/10.1016/j.jclinepi.2004.04.005

Richard, E., Reitz, C., Honig, L. H., Schupf, N., Tang, M. X., Manly, J. J., \& Luchsinger, J. A. (2013). Late-life depression, mild cognitive impairment, and dementia. JAMA Neurology, 70(3), 374-382. https://doi.org/ 10.1001/jamaneurol.2013.603

Salazar, C. R., Hoang, D., Gillen, D. L., \& Grill, J. D. (2020). Racial and ethnic differences in older adults' willingness to be contacted about Alzheimer's disease research participation. Alzheimer's \& Dementia: Translational Research \& Clinical Interventions, 6(1), e12023. https://doi.org/10.1002/ $\operatorname{trc} 2.12023$

Smirnoff, M., Wilets, I., Ragin, D. F., Adams, R., Holohan, J., Rhodes, R., Winkel, G., Ricci, E. M., Clesca, C., \& Richardson, L. D. (2018). A paradigm for understanding trust and mistrust in medical research: The Community VOICES study. AJOB Empirical Bioethics, 9(1), 39-47. https://doi.org/10.1080/ 23294515.2018.1432718 
U.S. Department of Health and Human Services. (2020). National Plan to Address Alzheimer's Disease: 2020 update. Accessed March 23, 2021 from https://aspe.hhs.gov/report/national-plan-address-alzhe imers-disease-2020-update

Watson, J. L., Ryan, L., Silverberg, N., Cahan, V., \& Bernard, M. A. (2014). Obstacles And Opportunities In Alzheimer's Clinical Trial Recruitment. Health Affairs, 33(4), 574-579. https://doi.org/10.1377/hlthaff. 2013.1314

Wendler, D., Kington, R., Madans, J., Wye, G. V., Christ-Schmidt, H., Pratt, L. A., \& Emanuel, E. (2005). Are Racial and Ethnic Minorities Less Willing to Participate in Health Research? PLOS Medicine, 3(2), e19. https://doi.org/10.1371/journal.pmed.0030019

Witham, M. D., \& McMurdo, M. E. T. (2007). How to get older people included in clinical studies. Drugs \& Aging, 24(3), 187-196. https://doi.org/10.2165/00002512-200724030-00002

Publisher's Note Springer Nature remains neutral with regard to jurisdictional claims in published maps and institutional affiliations.

Sadaf Arefi Milani, PhD, MPH is an Assistant Professor in the Department of Internal Medicine, Division of Geriatrics, at the University of Texas Medical Branch. Dr. Milani's research interests include epidemiology of aging, minority health and gender disparities, cognition and dementia, and pain and prescription opioid use.

Linda B. Cottler PhD, MPH, FACE is Dean's Professor in the Department of Epidemiology at the University of Florida and Associate Dean for Research in the College of Public Health and Health Professions. Dr. Cottler is the Founding Director of HealthStreet and her research interests include psychiatric epidemiology, diagnostic assessment development, community engagement research, substance abuse epidemiology, and global health.

Catherine W. Striley PhD, MSW,MPE is an Associate Professor in the Department of Epidemiology at the University of Florida. Dr. Striley is Co-Director of HealthStreet. Dr. Striley's research interests include psychiatric epidemiology, substance abuse epidemiology, health disparities, community engagement research, and research ethics. 